\title{
ADAM10 is overexpressed in human hepatocellular carcinoma and contributes to the proliferation, invasion and migration of HepG2 cells
}

\author{
SHAO YUAN ${ }^{1}$, SHI LEI ${ }^{2}$ and SHENGLI WU ${ }^{2}$ \\ Departments of ${ }^{1}$ Otorhinolaryngology and ${ }^{2}$ Hepatobiliary Surgery, The First Affiliated Hospital \\ of Xi'an Jiaotong University, Xi'an, Shaanxi 710061, P.R. China
}

Received May 24, 2013; Accepted July 1, 2013

DOI: $10.3892 / o r .2013 .2650$

\begin{abstract}
The overexpression of A disintegrin and metalloproteinase 10 (ADAM10) has been found to be closely associated with the development and progression of various types of tumors. However, ADAM10 expression in hepatocellular carcinoma (HCC) and its significance remain largely unknown. The present study aimed to investigate the expression of ADAM10 in human HCC and the effect of ADAM10 gene silencing by siRNA on the proliferation, invasion and migration of HepG2 human hepatoma cells. Immunohistochemistry was performed to examine the expression of ADAM10 in human HCC tissues and in the adjacent non-cancer tissues from 30 patients with HCC. RNA interference was used to knock down ADAM10 expression in HepG2 human hepatoma cells and the proliferation and migration as well as the invasive ability of the treated cells were observed in vitro. The expression of ADAM10 protein in HCC tissues was significantly higher when compared to that in adjacent non-tumor tissues $(\mathrm{P}<0.05)$. The high expression of ADAM10 in cancer was significantly correlated with clinical outcomes $(\mathrm{P}<0.05)$. Silencing of ADAM10 resulted in inhibition of proliferation and migration as well as invasion of HepG2 human hepatoma cells $(\mathrm{P}<0.05)$. These studies suggest that ADAM10 plays an important role in regulating proliferation, invasion and migration of HepG 2 cells. High expression of ADAM10 may be a valuable predictive factor for HCC prognosis, and ADAM10 is potentially an important therapeutic target for the prevention of tumor development and progression in HCC.
\end{abstract}

\section{Introduction}

Hepatocellular carcinoma (HCC) is one of the leading causes of cancer-related mortality worldwide (1), and despite the

Correspondence to: Dr Shengli Wu, Department of Hepatobiliary Surgery, The First Affiliated Hospital of Xi'an Jiaotong University, No. 277 Yanta West Road, Xi'an, Shaanxi 710061, P.R. China E-mail: victorywu2000@163.com

Key words: hepatocellular carcinoma, A disintegrin and metalloproteinase, siRNA, HepG2 cells extensive application of intensive surveillance programs, considerable therapeutic progress, and technological improvement observed over the past few years, prognosis of this tumor remains poor due to the high recurrence rate and metastatic potential of HCC cells, even when treatments have been considered potentially curative $(2,3)$. The incidence of intrahepatic or extrahepatic metastases is high in HCC with an infiltrative growth pattern according to clinicopathologic study (4). However, there is no effective chemotherapeutic agent which prevents recurrence and metastasis in HCC patients.

The A disintegrin and metalloproteinase (ADAM) family is a class of type I transmembrane proteins that contain two main structural domains: the disintegrin domain and the matrix metalloproteinase domain. Members of the ADAM family function as sheddases by cleaving type I and type II integral single membrane proteins to generate soluble forms of these proteins (5) and have been found to be involved in the etiologies of a variety of diseases and conditions (6-8). Members of the ADAM family can degrade the extracellular matrix (ECM) and control cell adhesion and movement through regulation of intercellular adhesion, protease activity and cell activities that are closely related to the metastasis of human tumors $(9,10)$. To date, 13 catalytically active ADAMs have been identified in the human genome $(11,12)$. One member of the ADAM family, A disintegrin and metalloproteinase 10 (ADAM10), has recently been reported to play important roles in cell migration, tumor development and metastasis by proteolytic shedding of cell surface proteins and has been demonstrated to be a positive regulator of cancer progression in renal cell carcinoma (13), pancreatic carcinoma (14), lung cancer (15), gastric carcinoma (16), oral squamous cell carcinoma (17) and melanoma (18). Proteolytically active ADAM10 acts as an ectodomain sheddase, which releases extracellular regions of membrane-bound proteins (e.g., adhesion molecules, growth factors, cytokines, chemokines and receptors). Through these actions it is able to sculpt the tumor microenvironment and modulate key processes involved in cancer progression, including cell proliferation, migration and angiogenesis (19). The emerging role of ADAM10 as a significant contributor to these pathologies has led to its intense interest as a potential drug target for tumor treatment (6). However, ADAM10 expression in HCC and its significance in HCC progression remain 
largely unknown. In the present study, we investigated the expression of ADAM10 in human HCC tissues and the adjacent non-cancerous tissues from $30 \mathrm{HCC}$ patients using immunohistochemistry. Moreover, the effects of ADAM10 gene silencing by siRNA on the proliferation, invasion and migration of HepG2 human hepatoma cells were observed in vitro.

\section{Materials and methods}

Ethics statement. The study was approved by the Ethics Committee of the First Affiliated Hospital of Xi'an Jiaotong University. All experiments were performed in accordance with the principles of the Declaration of Helsinki. All participants provided their written informed consent to participate in the study.

Patient information. A total of $30 \mathrm{HCC}$ patients who were treated with partial liver resection surgery at the Department of Hepatobiliary Surgery of the First Affiliated Hospital of Xi'an Jiaotong University from 2008 to 2011 were enrolled in this study. These patients included 24 males and 6 females, with a mean age of 53.16 years (range, $34-73$ years). The patients were pathologically diagnosed with $\mathrm{HCC}$ at histological grade I $(n=4)$, grade II $(n=19)$ and grade III $(n=7)$, and classified as stage I $(n=7)$, stage II $(n=17)$ and stage III $(n=6)$. Histological grading and staging were according to the modified nuclear grading scheme outlined by the Edmondson and Steiner and Okuda system, respectively $(20,21)$. No prior treatments (including chemotherapy or radiotherapy) were conducted before liver resection surgery.

Tissue samples and cell lines. The paraffin-embedded HCC tissues and the corresponding non-cancerous tissues from the 30 patients mentioned above were collected for pathological analysis. The human hepatoma cell line HepG2 was obtained from the Center of Biomedical Experimental Research at the Medical School, Xi'an Jiaotong University.

Immunohistochemical staining. Immunohistochemical staining was performed using streptavidin-peroxidase technique, and diaminobenzidine (DAB) was used as a chromogen. Rabbit polyclonal antibody against human ADAM10 protein was obtained from Santa Cruz Biotechnology, Inc. (Santa Cruz, CA, USA). The primary antibody for the negative control group was replaced with PBS. Five representative high-power fields (x400 magnification) for each tissue section were selected for histological evaluation. Two parameters, positive rate (PR) and staining intensity (SI), were used to describe the expression based on the extent and intensity of positively stained cells in the samples. PR is the percentage of positively stained cells in the tissues: $\leq 10 \%$ stained cells was defined as negative (scored as 0 ), 11-30\% stained cells was defined as positive with low frequency (scored as 1), 31-60\% stained cells was defined as positive with medium frequency (scored as 2), and $>60 \%$ stained cells was defined as positive with high frequency (scored as 3). SI is the ranked staining intensity of positively stained cells in the samples, which ranged from 0 to 3 , dim positive, mediate positive and strong positive, respectively. Since the expression of the protein was comprehensively evaluated based on both extent and inten- sity, the sum of both parameters provide the final scores for ADAM10 in each sample, in which the final score $<4$ was defined as low/negative expression, and the final score $\geq 4$ was defined as high expression.

Cell culture. HepG2 cells were cultured in Dulbecco's modified Eagle's medium (DMEM) (Invitrogen Life Technologies, Carlsbad, CA, USA) containing $10 \%$ fetal bovine serum (FBS) and $1 \%$ penicillin/streptomycin (Invitrogen Life Technologies) and incubated at $37^{\circ} \mathrm{C}$ in an atmosphere containing $5 \% \mathrm{CO}_{2}$.

siRNA transfection. For downregulation of endogenous ADAM10 expression, the following siRNA duplex (Aoke Biological Technology Co., Ltd., Shanghai, China) was used: 5'-AGACAUUAUGAAGGAUUAUTT-3'. As a negative control, the unspecific scrambled siRNA duplex (5'-AGGUAGUGUA AUCGCCUUGTT-3') was used.

At $24 \mathrm{~h}$ before transfection, $1 \times 10^{5}$ HepG 2 cells were seeded in 6-well plates. Transfection of siRNA was carried out using Lipofectamine 2000 (Invitrogen Life Technologies) and $10 \mathrm{nM}$ siRNA duplex/well. Transfection was performed as previously described (18). Specific silencing of targeted genes was confirmed by at least 3 independent experiments.

Four groups were established in this study: blank control group, Lipo2000 group (cells were treated with Lipofectamine 2000), control siRNA group (cells were treated with Lipofectamine 2000 plus the negative control siRNA), and ADAM10-siRNA group (cells were treated with Lipofectamine 2000 plus ADAM10 siRNA).

Real-time RT-PCR. Real-time RT-PCR for ADAM10 transcripts in HepG2 cells was carried out using the PrimeScript RT reagent kit following the manufacturer's instructions (Takara Bio, Inc., Shiga, Japan). ADAM10 gene-specific amplification was confirmed by PCR with specific primers (5'-CTGCCCAGCATCTGACCCTAA-3' and 5'-TTGCCATC AGAACTGGCACAC-3') and subjected to melting curve analysis. GAPDH was used as an internal control for standardization. All RT-PCR tests were performed in triplicate. The data were analyzed using the comparative $\mathrm{Ct}$ method.

Western blot analysis. Cells were washed twice with cold phosphate-buffered saline (PBS; $137 \mathrm{mM} \mathrm{NaCl}, 2.7 \mathrm{mM} \mathrm{KCl}$, $10 \mathrm{mM}$ sodium phosphate dibasic, $2 \mathrm{mM}$ potassium phosphate monobasic, $\mathrm{pH} 7.4)$ and lysed on ice in buffer $(150 \mathrm{mM} \mathrm{NaCl}$, $50 \mathrm{mM}$ Tris-HCl, 2 mM EDTA, 1\% NP-40, pH 7.4) containing protease inhibitors. Equal amounts of protein $(20 \mu \mathrm{g} / \mathrm{lane})$ from the cell lysates were electrophoresed under non-reducing conditions on $10 \%$ acrylamide gels. After SDS-PAGE, proteins were transferred to a polyvinylidene difluoride membrane. The membrane was incubated for $2 \mathrm{~h}$ in PBS plus $0.1 \%$ Tween-20 and $5 \%$ nonfat skim milk to block nonspecific binding. Subsequently, the membrane was incubated for $2 \mathrm{~h}$ with an antibody against ADAM10 (R\&D Systems, Minneapolis, MN, USA). After washing, proteins were visualized using an ECL detection kit with the appropriate HRP-conjugated secondary antibody (Amersham Pharmacia Biotech, Piscataway, NJ, USA). The membranes were stripped and probed with monoclonal antibodies for GAPDH for the loading control as per standard protocols. 


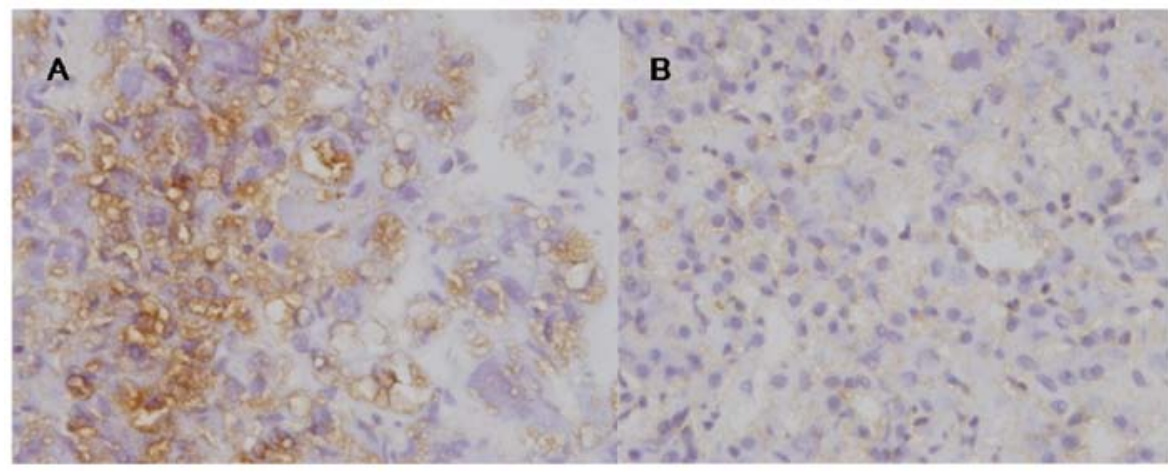

Figure 1. Immunohistochemical staining of ADAM10 protein of HCC and the corresponding non-cancerous tissues of magnification x200. (A) Strong immunostaining for ADAM10 protein in HCC tissue. (B) ADAM10 protein expression is absent in non-cancerous liver tissue. HCC, hepatocellular carcinoma; ADAM10, A disintegrin and metalloproteinase 10.

Proliferation assay. The 3-[4,5-dimethylthiazol-2-yl]-2,5-diphenyltetrazolium bromide (MTT) (Sigma Corporation, USA) colorimetric assay was used to screen for cell proliferation. Briefly, HepG2 cells were seeded into eight 96 -well plates at a density of $2 \times 10^{3}$ cells/well and incubated in RPMI-1640 medium for 24, 48, 72 and $96 \mathrm{~h}$ after treatment, respectively. Twenty microliters of MTT $(5 \mathrm{mg} / \mathrm{ml})$ was added into each well, and the cell culture was continued for $4 \mathrm{~h}$. After aspiration of the medium, the cells were lysed with DMSO (Sigma Corporation). The absorbance was measured using a microplate reader at a wavelength of $490 \mathrm{~nm}$. The cell growth curve was plotted with OD values as the ordinate against time as the abscissa. The experiment was repeated 3 times.

Soft agar colony formation assay. Briefly, $4 \times 10^{3}$ HepG2 cells were mixed with $0.5 \%$ top agar and seeded on 24-well plates with $1 \%$ base agar. These cells were then cultured in an incubator with $5 \% \mathrm{CO}_{2}$ and $95 \%$ humidity at $37^{\circ} \mathrm{C}$ for 10 days. Finally, the cell colonies in soft agar were photographed and counted by a microscope. All of the experiments were independently repeated at least 3 times.

Cell migration assay. The effect of ADAM10 knockdown on HepG2 cell migration was measured as the ability of cells to migrate through Transwell filters $(6.5-\mathrm{mm}$ diameter, $5-\mu \mathrm{m}$ pore size). Transwell filters were coated with Matrigel for $1.5 \mathrm{~h}$ before adding the cells. At $24 \mathrm{~h}$ after the siRNA transfection, cells were detached by trypsinization, and $1 \times 10^{5}$ cells were seeded into Transwell filters in $100 \mathrm{ml}$ of starvation medium. Growth medium $(500 \mathrm{ml})$ was placed in the lower compartment, and the cells were left to migrate for $24 \mathrm{~h}$. Non-migratory cells were removed by a cotton swab, and the transmigrated cells at the backside of the filter were stained with Giemsa. HepG2 cells on each filter were counted at $x 400$ magnification to quantitate HepG2 cell migration. Images of 3 random fields from 3 replicate wells were obtained. Migration was determined as the mean of the cells that had migrated per $x 400$ field and was expressed as a percentage of the blank control.

In vitro invasion assay. HepG2 cell invasive behavior was evaluated using 24 -well Transwell units with $8-\mu$ m porosity polycarbonate filters. The filters were coated with $50 \mu \mathrm{l}$ of $8 \mathrm{mg} / \mathrm{ml}$ reconstituted basement membrane substance
(Matrigel; BD Biosciences, San Diego, CA, USA). The coated filters were air-dried at $4^{\circ} \mathrm{C}$ prior to the addition of the cells. The basement membrane was hydrated with $50 \mu$ l serum-free RPMI-1640 medium 30 min before use. The cells were digested with trypsin, and the cell density was adjusted to $1 \times 10^{6} / \mathrm{ml}$ using serum-free RPMI-1640 medium. A total of $200 \mu \mathrm{l}$ of cell suspension was added into each upper Transwell chamber, and $600 \mu \mathrm{l}$ of RPMI-1640 medium containing 5\% fetal bovine serum was added into the lower chamber. There were three duplicates for each cell group. The cells were then incubated for $24 \mathrm{~h}$ in a humidified atmosphere of $5 \% \mathrm{CO}_{2}$ at $37^{\circ} \mathrm{C}$. Cells were fixed with methanol and stained with Giemsa. Cells on the upper surface of the filter were removed by wiping with a cotton swab, and invasion was determined by counting the cells that migrated to the lower side of the filter with optical microscopy at x400 magnification. A total of 5 visual fields at the center and in the surrounding areas was counted, and the average was calculated (22). The experiment was repeated 3 times.

Data and statistical analysis. All data are presented as the means \pm standard error of the mean. Statistical analysis was performed using SPSS 16.0 software. Differences among groups were tested by one-way analysis of variance (ANOVA). A P-value $<0.05$ was considered to indicate a statistically significant result.

\section{Results}

Expression of ADAMIO protein in paired HCC and corresponding non-cancerous tissue samples. ADAM10 was detected in 22 out of $30(73.33 \%)$ HCC tissues and in only 1 out of the 30 (3.33\%) corresponding non-cancerous tissues (Fig. 1). The frequency of ADAM10 in HCC tissues was significantly higher than that in the non-cancerous tissues $(\mathrm{P}<0.05)$.

Increase in ADAM10 expression correlates with worse prognosis and shorter survival of patients after surgery. All of the 30 patients were completely followed up. During follow-up, 13 patients died, whereas 17 patients were alive at the end of the study. The median follow-up period was 28 months (3-45 months). The median survival time was 22.3 months (95\% CI, 20.09-26.46). We compared the expression of 
Table I. Correlation between the expression of ADAM10 in the 30 cases of HCC tissues and multiple clinicopathological features of the corresponding patients.

\begin{tabular}{|c|c|c|c|c|}
\hline \multirow[b]{2}{*}{ Features } & \multirow[b]{2}{*}{ Case } & \multicolumn{2}{|c|}{$\begin{array}{c}\text { Expression of } \\
\text { ADAM10 }\end{array}$} & \multirow[b]{2}{*}{ P-value } \\
\hline & & - & + & \\
\hline \multicolumn{5}{|l|}{ Gender } \\
\hline Female & 6 & 2 & 4 & 0.680 \\
\hline Male & 24 & 6 & 18 & \\
\hline \multicolumn{5}{|l|}{ Age (years) } \\
\hline$<53.16$ & 14 & 6 & 8 & 0.061 \\
\hline$\geq 53.16$ & 16 & 2 & 14 & \\
\hline \multicolumn{5}{|c|}{ HBV infection } \\
\hline No & 4 & 1 & 3 & 0.935 \\
\hline Yes & 26 & 7 & 19 & \\
\hline \multicolumn{5}{|c|}{ Alcohol history } \\
\hline No & 13 & 5 & 8 & 0.201 \\
\hline Yes & 17 & 3 & 14 & \\
\hline \multicolumn{5}{|c|}{ Smoking history } \\
\hline No & 22 & 5 & 17 & 0.418 \\
\hline Yes & 8 & 3 & 5 & \\
\hline \multicolumn{5}{|c|}{ Clinical stage of disease } \\
\hline I & 7 & 2 & 5 & 0.889 \\
\hline II & 17 & 4 & 13 & \\
\hline III & 6 & 2 & 4 & \\
\hline \multicolumn{5}{|c|}{ Histological grade } \\
\hline I & 4 & 2 & 2 & 0.488 \\
\hline II & 19 & 4 & 15 & \\
\hline III & 7 & 2 & 5 & \\
\hline \multicolumn{5}{|c|}{ Microvascular invasion } \\
\hline Presence & 12 & 4 & 8 & 0.396 \\
\hline Absence & 18 & 4 & 14 & \\
\hline \multicolumn{5}{|c|}{ Clinical outcome } \\
\hline Alive & 17 & 7 & 10 & 0.040 \\
\hline Deceased & 13 & 1 & 12 & \\
\hline
\end{tabular}

-, Low/negative expression of ADAM10 in cancer tissues; + , high expression of ADAM10 in cancer tissues. ADAM10, A disintegrin and metalloproteinase 10; HCC, hepatocellular carcinoma.

ADAM10 in the patients according to different gender, age, status of HBV infection, history of alcohol consumption and smoking, disease stage, histological differentiation, microvascular invasion and clinical outcome. The levels of ADAM10 expression did not significantly vary between males and females, elderly and younger, and HBV infected and uninfected patients. No significant difference was identified between patients with and without a history of alcohol consumption or smoking. The expression levels of ADAM10 did correlate with the clinical outcomes although the level did not vary significantly among HCC samples at different clinical stages, degree of histological differentiation and microvascular invasion. It

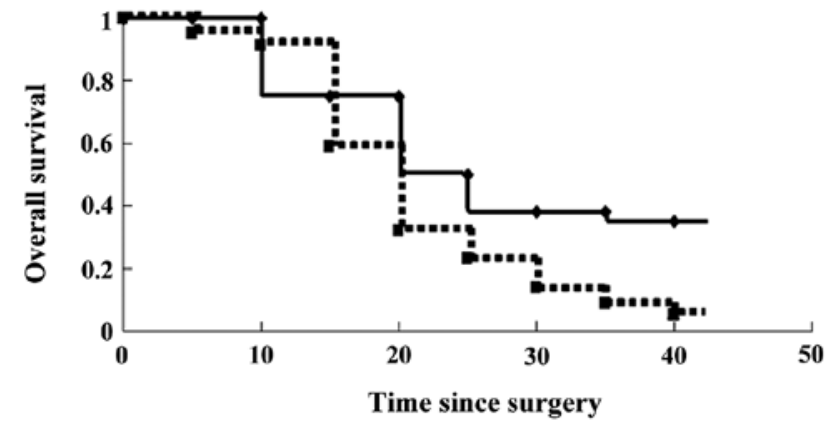

.... ADAM10 high group — ADAM10 low/negative group

Figure 2. Kaplan-Meier curves based on ADAM10 expression. Kaplan-Meier estimates for overall survival (months) based on ADAM10 status. ADAM10, A disintegrin and metalloproteinase 10 .

was observed that 12 of the 13 HCC patients who passed away had tumors with high ADAM10 expression. This percentage was significantly higher than the percentage in the living HCC patients ( 92.31 vs. $58.82 \%, \mathrm{P}=0.040$, Table I). Using the Kaplan-Meier method, the overall survival rates were estimated for the 30 patients. Overall survival was significantly shorter in the ADAM10 high expression group than that in the ADAM10 low/negative expression group ( $\mathrm{p}=0.044)$ (Fig. 2). This result implies that high expression of ADAM10 may be a valuable predictive factor for $\mathrm{HCC}$ prognosis.

Knockdown of ADAM10 in HepG2 cells. The expression of ADAM10 was examined by real-time RT-PCR and western blotting to validate the silencing efficiency of the target gene after RNAi. Stable ADAM10 siRNA-transfected HepG2 cells (ADAM10-siRNA) and a mock-transfected control cell line (control siRNA) were established as described above. Compared to the parental HepG2 cells and control siRNA cells, both mRNA and protein expression of ADAM10 was significantly reduced in the ADAM10 siRNA cells at $24 \mathrm{~h}$ after siRNA transfection (all $\mathrm{P}<0.05$; Fig. $3 \mathrm{~A}$ and $\mathrm{B}$ ), which persisted for at least $96 \mathrm{~h}$ (data not shown).

Gene silencing of ADAM10 reduces cell proliferation and cell colony formation in HepG2 cells. To examine whether the knockdown of ADAM10 expression had any affect on cell growth, an MTT cell proliferation assay was performed. Compared to the blank control group, the Lipo2000 group, and the control siRNA group cells, ADAM10-siRNA group cells showed decreased cell proliferation, supporting the role of ADAM10 in cell growth in HepG2 cells (P<0.05, Fig. 4). In addition, the affect of gene silencing of ADAM10 on the cell colony formation of HepG2 cells was also investigated by a soft agar colony formation assay. The results indicated that the cell colony number significantly decreased in the ADAM10-siRNA group compared to the blank control group, Lipo2000 group, and control siRNA group $(\mathrm{P}<0.05$, Fig. 5A and B).

Gene silencing of ADAM10 reduces cell migration in HepG2 cells. The effect of gene silencing of ADAM10 on 

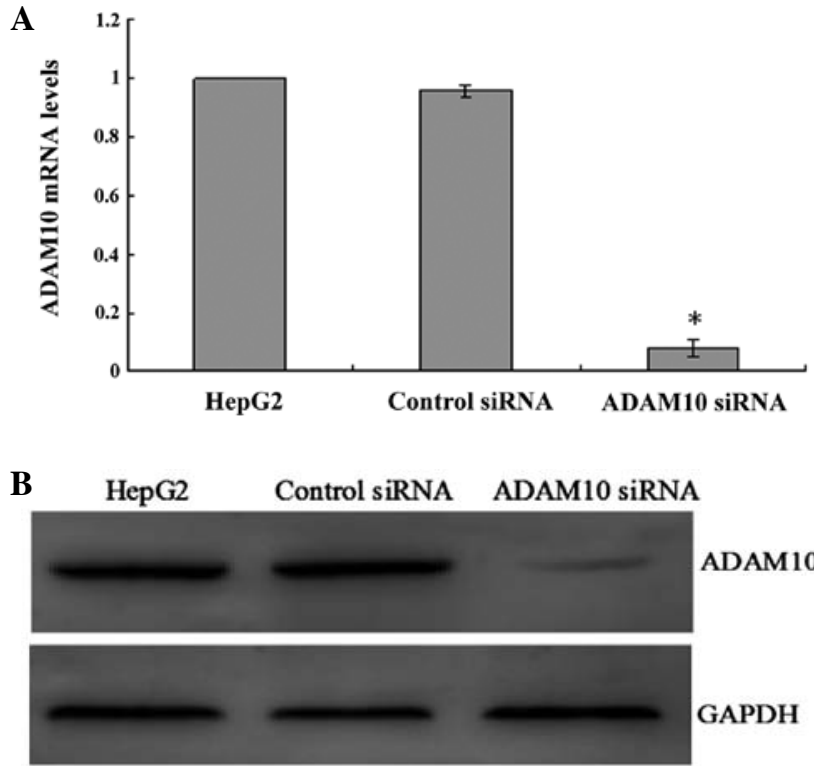

Figure 3. Knockdown of ADAM10 expression in HepG2 cells at $24 \mathrm{~h}$ after siRNA transfection. (A) ADAM10 mRNA levels were determined by real-time RT-PCR. Relative fold induction for ADAM10 mRNA (means $\pm \mathrm{SD}$ ) in the mock- and ADAM10 siRNA-transfected HepG2 cells is presented relative to the expression in the parental HepG2 cells $($ $P<0.05$ compared with the parental HepG2 cells). (B) Western blot analysis for ADAM10 protein expression in the indicated cell lines. GAPDH was used as a loading control. Parental HepG2 cells, control siRNA (mock-transfected control), and ADAM10-siRNA represent the 3 different clones, respectively. ADAM10, A disintegrin and metalloproteinase 10.

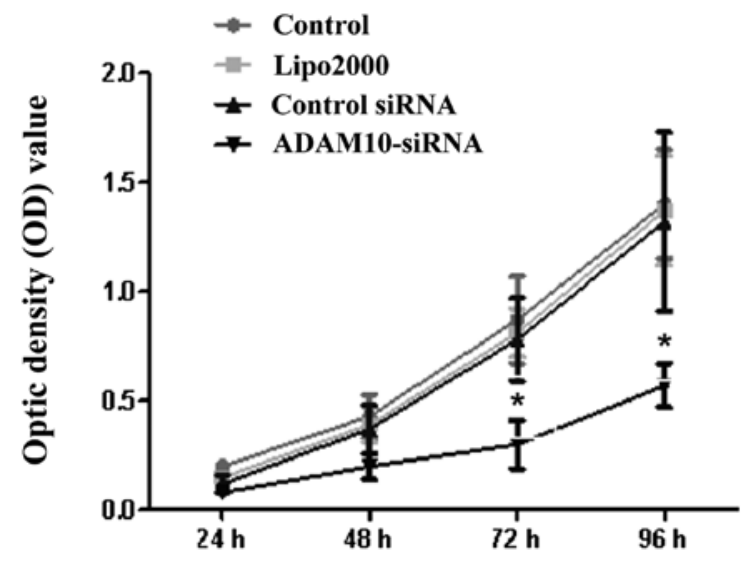

Figure 4. Gene silencing of ADAM10 reduces cell proliferation in HepG2 cells. Cell proliferation was analyzed using the MTT assay. Cells were monitored for $96 \mathrm{~h}$, and the average OD490 ( \pm SD) for each cell line is shown Cells transfected with ADAM10 siRNA (ADAM10-siRNA) showed reduced cell growth relative to the blank control group, Lipo2000 group, and control siRNA group cells, at 72 and $96 \mathrm{~h}$ after treatment, respectively ( ${ }^{*} \mathrm{P}<0.05$ compared with the blank control group, Lipo2000 group and control siRNA group, respectively). ADAM10, A disintegrin and metalloproteinase 10.

the cell migration ability of HepG2 cells was investigated by Transwell invasion assay (Fig. 6A). The results indicated that ADAM10 siRNA cells had a significantly reduced ability to pass through the basement membrane when compared to the cells in the other 3 groups (all $\mathrm{P}<0.05$; Fig. 6B). These data support the notion that ADAM10 expression is essential for cell migration.
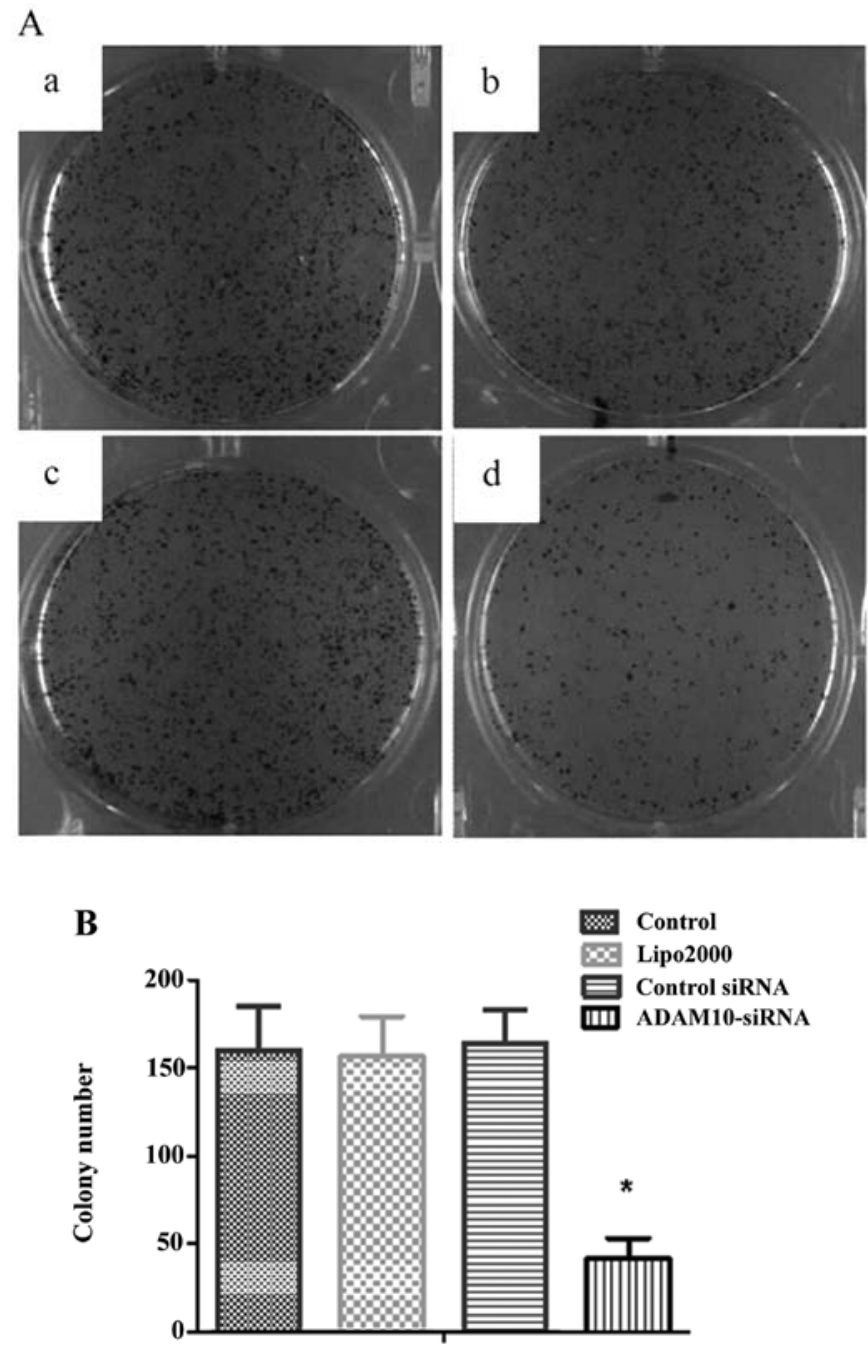

Figure 5. Gene silencing of ADAM10 reduces cell colony formation in HepG2 cells. (A) ADAM10 knockdown by siRNA reduced cell colony formation, as shown by the representative dishes of colony formation of cells in the blank control group (dish a), Lipo2000 group (dish b), control siRNA group (dish c), and ADAM10-siRNA group (dish d). (B) The mean numbers of colonies of 3 independent experiments (means \pm SD) are indicated by the histograms ( $\mathrm{P}<0.05$ compared with the blank control group, Lipo2000 group and control siRNA group, respectively). ADAM10, A disintegrin and metalloproteinase 10.

Gene silencing of ADAM10 reduces the invasive ability of HepG2 cells. The invasive ability of HepG2 cells was investigated using a Matrigel invasion assay. The invasion assay results indicated that HepG2 cells had a significantly lower ability to pass through the basement membrane compared to the cells in the other three groups (all $\mathrm{P}<0.05$; Fig. 7A and $\mathrm{B}$ ). These results indicate that ADAM10 plays a key role in hepatocarcinoma cell invasion.

\section{Discussion}

Various members of the ADAM family including ADAM10 have been shown to be overexpressed in malignant tumors and may be related to the biological behavior. For example, downregulation of ADAM10 has been shown to suppress cell proliferation and decrease the metastatic potential of adenoid cystic carcinoma cells (23). We hypothesized that the 

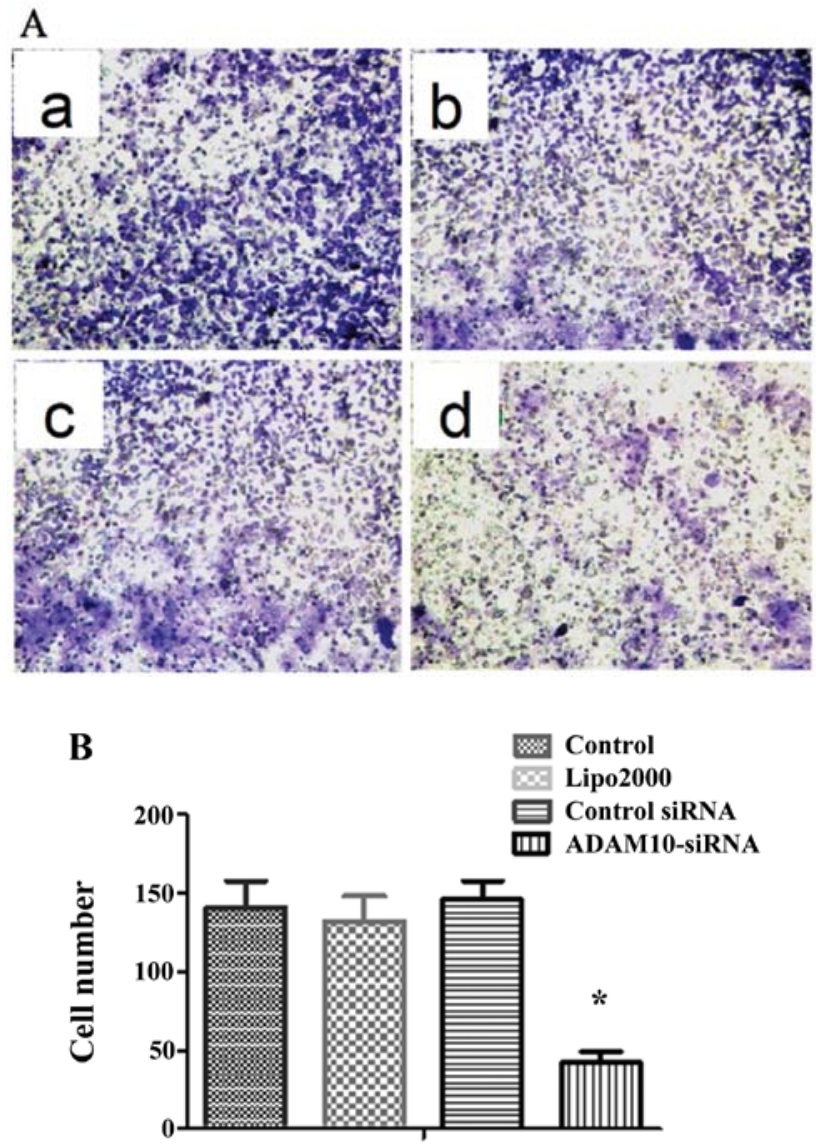

Figure 6. Gene silencing of ADAM10 reduces cell migration in HepG2 cells. (A) A Matrigel Transwell invasion assay was used to test the migration ability of the cells (a, blank control group; b, Lipo2000 group; c, control siRNA group and d, ADAM10-siRNA group) to pass through the basement membrane. (B) Values represent the cell number (means \pm SD) per visible field ("P $<0.05$ compared with the blank control group, Lipo2000 group and control siRNA group, respectively). ADAM10, A disintegrin and metalloproteinase 10 .

expression of ADAM10 is increased in HCC and their downregulation of ADAM10 may influence the biological behavior of HepG2 cells. However, previous reports that may relate to this hypothesis are scarce. Thus, the purpose of this study was to evaluate the expression of ADAM10 in HCC tissues and adjacent non-tumor tissues and to analyze the relationship between the gene silencing of ADAM10 and the proliferation, invasion and migration capability of HepG2 cells in vitro.

In this study, we characterized the expression of ADAM10 in HCC tissues. Immunohistochemical analysis indicated that ADAM10 expression was significantly elevated in HCC tissues when compared to that in the adjacent non-tumor tissues, suggesting that ADAM10 may be associated with hepatocarcinogenesis. Further survival analysis demonstrated that the high expression of ADAM10 in HCC was significantly correlated with worse prognosis and shorter survival of patients following surgery, suggesting that high expression of ADAM10 may be a valuable predictive factor for HCC prognosis.

Currently, surgery is the preferred treatment method for liver cancer, yet the 5-year survival rate remains extremely low due to the high recurrence rate and the metastatic potential of HCC cells. It is reported that up to $70 \%$ of the patients show relapse within 5 years after curative resection (24). Autopsy
A
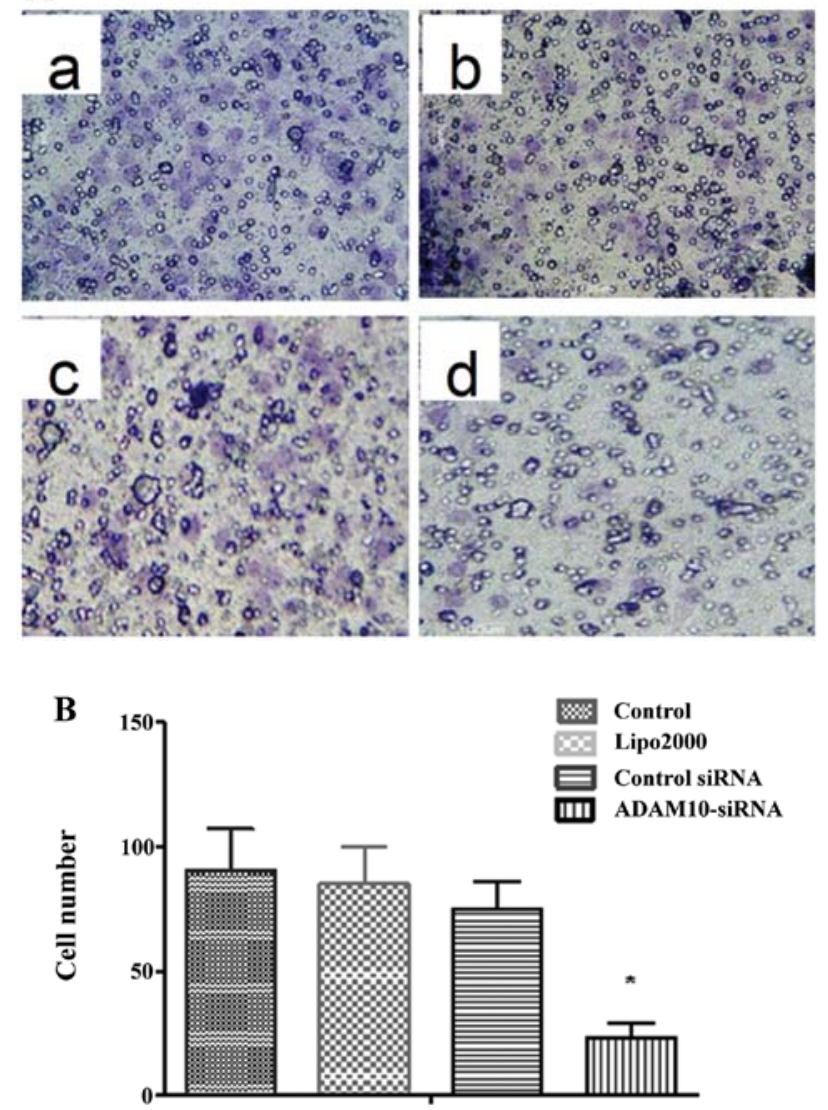

Figure 7. Gene silencing of ADAM10 reduces the invasive ability of HepG2 cells. (A) A Matrigel Transwell invasion assay was used to test the ability of HepG2 cells to invade the filter membrane (a, blank control group; b, Lipo2000 group; c, control siRNA group and d, ADAM10-siRNA group). (B) Values represent the cell number (means \pm SD) per visible field ( $(\mathrm{P}<0.05$ compared with blank control group, Lipo2000 group and control siRNA group, respectively). ADAM10, A disintegrin and metalloproteinase 10.

studies confirm that nearly one-third of all HCC patients have lymph node metastasis, which is the leading cause for distant metastasis and mortality (25). In other studies, overexpression of ADAM10 has been demonstrated to be a potential prognostic indicator for high risk of recurrence and metastasis $(18,23,26)$. Based on these data, it is reasonable to speculate that ADAM10 may play a role in HCC growth and metastasis.

To provide evidence supporting this supposition, we investigated the effects of ADAM10 silencing on the proliferation, invasion and migration of human hepatoma HepG2 cells in vitro. The expression of ADAM10 was specifically knocked down in the human HepG2 cell line using RNAi. Downregulation of ADAM10 resulted in suppression of tumor cell proliferation, which strongly supports that ADAM10 is involved in the process of tumor development. Our data are in line with previous reports showing that ADAM10 expression is correlated with the proliferation of tumor cells. Ko et al (17) demonstrated that the expression of ADAM10 was correlated with increased growth of oral squamous cell carcinoma cells. Arima et al (27) confirmed that suppression of ADAM10 expression leads to a significant decrease in prostate cancer cell growth. The effect of ADAM10 on tumor cell growth may be related to its protease activity. ADAM10 can cleave 
several critical transmembrane molecules including amyloid precursor protein $(28,29)$. It must be noted that soluble amyloid precursor protein has been related to the growth of several types of cells $(30,31)$, which suggests that ADAM10 may influence the proliferation of HepG 2 cells via amyloid precursor protein shedding.

In addition, in the present study, the transfection of ADAM10 siRNA resulted in a significant reduction in cellular invasion and migration of HepG2 cells, which strongly indicates that ADAM10 is involved in the process of HCC metastasis. Our finding is in agreement with previous reports on the functional roles of ADAM10. Biological behaviors of cancer cells are regulated by multiple growth factors and cytokines. Many cytokines and growth factors involved in this process are synthesized as membrane bound proforms which undergo proteolytic shedding for activation. ADAM10 is one of the representative proteases that mediate proteolytic shedding, and this process appears to be involved in the pathophysiology of various diseases such as cancer (9). Tumor metastasis is dependent on the ability of the tumor to degrade the surrounding ECM and reduced cell adhesion. Studies have demonstrated that ADAM10 can cleave and remodel ECM proteins such as CD44. Pan et al (32) found that in the pituitary adenoma cell line, AtT-20, ADAM10 facilitated cell migration through modulation of CD44 and L1 cleavage. In another study, Anderegg et al (33) showed that ADAM10 is the predominant protease involved in the constitutive shedding of endogenous CD44 from melanoma cells. Multiple cell signaling pathways are responsible for cellular proliferation and migration (34). ADAM10 may also promote tumor metastasis by influencing cell-cell signaling. E-cadherin is a transmembrane molecule which functions as an adhesion molecule. Increased expression of ADAM10 may lead to elevated shedding of E-cadherin and loss of cell-cell contact (18). Expression levels of several components of the Notch pathway, which can be cleaved by ADAM10, were upregulated in melanomas compared with common melanocytic nevi (35). Guo et al (15) presented further evidence that ADAM10 promotes non-small cell lung cancer (NSCLC) cell migration and invasion via the activation of the Notch1 signaling pathway. In another study, Gutwein et al (36) demonstrated that the release of cell-associated adhesion molecules such as L1 may be relevant to promote cell migration, and L1 release in AR breast carcinoma cells is mediated by ADAM10. Taken together, ADAM10 is able to modulate a variety of cellcell and cell-ECM interactions and can consequently digest the basement membrane, facilitate cell migration and promote tumor metastasis. Importantly, in the present study, we discovered that downregulation of ADAM10 via ADAM10-specific siRNA significantly inhibited the proliferation, invasion and migration capability of HepG2 cells, which suggests that ADAM10 is a promising new therapeutic target for the treatment of HCC. In the future, studies using undifferentiated and aggressive hepatocarcinoma cell lines with metastatic potential, such as MHCC97-H and SNU398 may be used to further reveal the role of ADAM10 in hepatocarcinogenesis and HCC progression.

In conclusion, our data revealed that ADAM10 expression in HCC tissues was significantly higher than that in adjacent non-tumor tissues. High expression of ADAM10 may be a valu- able predictive factor for HCC prognosis. Reduced ADAM10 expression not only impacted cell proliferation, but also decreased the metastatic potential of HepG2 cells, indicating that ADAM10 may participate in hepatocarcinogenesis and HCC progression. Thus, ADAM10 is a potential therapeutic target for the treatment of HCC.

\section{Acknowledgements}

The authors thank Dr Zuoren Wang for review of the manuscript and the helpful comments.

\section{References}

1. El-Serag HB: Hepatocellular carcinoma. N Engl J Med 365: 1118-1127, 2011.

2. Bruix J and Sherman M; American Association for the Study of Liver Diseases: Management of hepatocellular carcinoma: an update. Hepatology 53: 1020-1022, 2011.

3. Wang N, Feng Y, Lau EP, et al: F-actin reorganization and inactivation of rho signaling pathway involved in the inhibitory effect of Coptidis Rhizoma on hepatoma cell migration. Integr Cancer Ther 9: 354-364, 2010.

4. Kanai T, Hirohashi S, Upton MP, et al: Pathology of small hepatocellular carcinoma. A proposal for a new gross classification. Cancer 60: 810-819, 1987.

5. Saftig P and Reiss K: The 'A Disintegrin And Metalloproteases' ADAM10 and ADAM17: novel drug targets with therapeutic potential? Eur J Cell Biol 90: 527-535, 2011.

6. Crawford HC, Dempsey PJ, Brown G, Adam L and Moss ML: ADAM10 as a therapeutic target for cancer and inflammation. Curr Pharm Des 15: 2288-2299, 2009.

7. Postina R, Schroeder A, Dewachter I, et al: A disintegrin-metalloproteinase prevents amyloid plaque formation and hippocampal defects in an Alzheimer disease mouse model. J Clin Invest 113: 1456-1464, 2004.

8. Naus S, Blanchet MR, Gossens K, Zaph C, Bartsch JW, McNagny KM and Ziltener HJ: The metalloprotease-disintegrin ADAM8 is essential for the development of experimental asthma. Am J Respir Crit Care Med 181: 1318-1328, 2010.

9. Murphy G: The ADAMs: signalling scissors in the tumour microenvironment. Nat Rev Cancer 8: 929-941, 2008.

10. Lu X, Lu D, Scully M and Kakkar V: ADAM proteins - therapeutic potential in cancer. Curr Cancer Drug Targets 8: 720-732, 2008.

11. Becherer JD and Blobel CP: Biochemical properties and functions of membrane-anchored metalloprotease-disintegrin proteins (ADAMs). Curr Top Dev Biol 54: 101-123, 2003.

12. Huang J, Bridges LC and White JM: Selective modulation of integrin-mediated cell migration by distinct ADAM family members. Mol Biol Cell 16: 4982-4991, 2005

13. Doberstein K, Pfeilschifter J and Gutwein P: The transcription factor PAX2 regulates ADAM10 expression in renal cell carcinoma. Carcinogenesis 32: 1713-1723, 2011.

14. Gaida MM, Haag N, Günther F, et al: Expression of A disintegrin and metalloprotease 10 in pancreatic carcinoma. Int J Mol Med 26: 281-288, 2010.

15. Guo J, He L, Yuan P, et al: ADAM10 overexpression in human non-small cell lung cancer correlates with cell migration and invasion through the activation of the Notch1 signaling pathway. Oncol Rep 28: 1709-1718, 2012.

16. Wang YY, Ye ZY, Li L, Zhao ZS, Shao QS and Tao HQ: ADAM 10 is associated with gastric cancer progression and prognosis of patients. J Surg Oncol 103: 116-123, 2011.

17. Ko SY, Lin SC, Wong YK, Liu CJ, Chang KW and Liu TY: Increase of disintergin metalloprotease 10 (ADAM10) expression in oral squamous cell carcinoma. Cancer Lett 245: 33-43, 2007.

18. Lee SB, Schramme A, Doberstein K, et al: ADAM10 is upregulated in melanoma metastasis compared with primary melanoma. J Invest Dermatol 130: 763-773, 2010.

19. Turner SL, Blair-Zajdel ME and Bunning RA: ADAMs and ADAMTSs in cancer. Br J Biomed Sci 66: 117-128, 2009.

20. Pawlik TM, Gleisner AL, Anders RA, Assumpcao L, Maley W and Choti MA: Preoperative assessment of hepatocellular carcinoma tumor grade using needle biopsy: implications for transplant eligibility. Ann Surg 245: 435-442, 2007. 
21. Okuda K, Ohtsuki T, Obata H, et al: Natural history of hepatocellular carcinoma and prognosis in relation to treatment. Study of 850 patients. Cancer 56: 918-928, 1985.

22. Yu Y, Chen W, Zhang Y, Hamburger AW, Pan H and Zhang Z: Suppression of salivary adenoid cystic carcinoma growth and metastasis by ErbB3 binding protein Ebp1 gene transfer. Int J Cancer 120: 1909-1913, 2007.

23. Xu Q, Liu X, Chen W and Zhang Z: Inhibiting adenoid cystic carcinoma cell growth and metastasis by blocking the expression of ADAM 10 using RNA interference. J Transl Med 8: 136, 2010.

24. Llovet JM, Schwartz M and Mazzaferro V: Resection and liver transplantation for hepatocellular carcinoma. Semin Liver Dis 25: 181-200, 2005.

25. Zhu W, Owusu L, Zang S, Zhang Y, Xin Y and Yan C: GRP78 and GAL3, differentially regulated by lymph node homogenates, as potential biomarkers for lymph node metastasis in mouse hepatocellular carcinoma cells. Oncol Lett 4: 1374-1378, 2012.

26. Gavert N, Conacci-Sorrell M, Gast D, Schneider A, Altevogt P, Brabletz T and Ben-Ze'ev A: L1, a novel target of $\beta$-catenin signaling, transforms cells and is expressed at the invasive front of colon cancers. J Cell Biol 168: 633-642, 2005.

27. Arima T, Enokida H, Kubo H, et al: Nuclear translocation of ADAM-10 contributes to the pathogenesis and progression of human prostate cancer. Cancer Sci 98: 1720-1726, 2007.

28. Jorissen E, Prox J, Bernreuther C, et al: The disintegrin/metalloproteinase ADAM10 is essential for the establishment of the brain cortex. J Neurosci 30: 4833-4844, 2010.

29. Jacobsen KT, Adlerz L, Multhaup G and Iverfeldt K: Insulin-like growth factor-1 (IGF-1)-induced processing of amyloid- $\beta$ precursor protein (APP) and APP-like protein 2 is mediated by different metalloproteinases. J Biol Chem 285: 10223-10231, 2010.
30. Venkataramani V, Rossner C, Iffland L, et al: Histone deacetylase inhibitor valproic acid inhibits cancer cell proliferation via down-regulation of the alzheimer amyloid precursor protein. J Biol Chem 285: 10678-10689, 2010.

31. Fan X, Liu Y, Jiang J, et al: miR-20a promotes proliferation and invasion by targeting APP in human ovarian cancer cells. Acta Biochim Biophys Sin 42: 318-324, 2010.

32. Pan $\mathrm{Y}$, Han $\mathrm{C}$, Wang $\mathrm{C}$, et al: ADAM10 promotes pituitary adenoma cell migration by regulating cleavage of CD44 and L1. J Mol Endocrinol 49: 21-33, 2012.

33. Anderegg U, Eichenberg T, Parthaune T, et al: ADAM10 is the constitutive functional sheddase of CD44 in human melanoma cells. J Invest Dermatol 129: 1471-1482, 2009.

34. DeCicco-Skinner KL, Nolan SJ, Deshpande MM, Trovato EL, Dempsey TA and Wiest JS: Altered prostanoid signaling contributes to increased skin tumorigenesis in Tpl2 knockout mice. PLoS One 8: e56212, 2013.

35. Massi D, Tarantini F, Franchi A, et al: Evidence for differential expression of Notch receptors and their ligands in melanocytic nevi and cutaneous malignant melanoma. Mod Pathol 19: 246-254, 2006.

36. Gutwein P, Oleszewski M, Mechtersheimer S, Agmon-Levin N, Krauss K and Altevogt P: Role of Src kinases in the ADAMmediated release of $\mathrm{L} 1$ adhesion molecule from human tumor cells. J Biol Chem 275: 15490-15497, 2000 